\section{sciendo}

DOI: $10.2478 /$ awutm-2018-0016
Analele Universitătii de Vest,

Timişoara

Seria Matematică - Informatică

LVI, 2, (2018), 71- 80

\title{
Iterated function systems consisting of continuous functions satisfying Banach's orbital condition
}

Radu Miculescu, Alexandru Mihail, and Irina Savu

Dedicated to Professor Mihail Megan on the occasion of his 70th birthday

\begin{abstract}
We introduce the concept of iterated function system consisting of continuous functions satisfying Banach's orbital condition and prove that the fractal operator associated to such a system is weakly Picard. Some examples are provided.
\end{abstract}

AMS Subject Classification (2000). 28A80, 37C70, 54H20

Keywords. iterated function system, Banach's orbital condition, fractal operator, weakly Picard operator, attractor

\section{Introduction}

As part of the motivation of Definition 3.1, let us recall that, on the one hand, in the development of fixed point theory by finding more general conditions which guarantee the existence (and uniqueness) of fixed points, an important role is played by the so called Banach orbital condition. It has been studied by several mathematicians (see [3], [10], [13], [21]- [22] and [25]). For example we have the following:

Theorem 1.1. (see [10]). Let $(X, d)$ be a metric space and $f: X \rightarrow X$ such that there exists $C \in[0,1)$ having the property that

$$
d\left(f(x), f^{[2]}(x)\right) \leq C d(x, f(x)),
$$


for every $x \in X$. Then:

i) For every $x_{0} \in X$, the sequence $\left(f^{[n]}\left(x_{0}\right)\right)_{n \in \mathbb{N}}$ is Cauchy.

ii) If, in addition, $(X, d)$ is complete and the function $g: X \rightarrow X$, given by $g(x)=d(x, f(x))$ for every $x \in X$, is lower semi-continuous at a point $\alpha \in\left\{f^{[n]}\left(x_{0}\right) \mid n \in \mathbb{N}\right\}^{\prime}$, then $f(\alpha)=\alpha$.

On the other hand, we recall that using the concept of iterated function system introduced by J. Hutchinson (see [11]) one can obtain a significant class of fractals. Consequently various authors extended this notion (see [1]-[2], [4]-[9], [12], [14]-[20], [23]- [24] and [26] and the references therein).

By combining these two lines of research, we introduce the concept of iterated function system consisting of continuous functions satisfying Banach's orbital condition and prove that the fractal operator associated to such a system is weakly Picard. In particular we obtain that a continuous function $f: X \rightarrow$ $X$, where $(X, d)$ is a complete metric space, which satisfies Banach's orbital condition is weakly Picard. Two examples are provided.

\section{Preliminaries}

\section{Notations and terminology}

Given two sets $X$ and $Y$, we consider the functions $\pi_{1}: X \times Y \rightarrow X$ given by $\pi_{1}(x, y)=x$ for every $(x, y) \in X \times Y$ and $\pi_{2}: X \times Y \rightarrow Y$ given by $\pi_{2}(x, y)=y$ for every $(x, y) \in X \times Y$.

Given a set $X$, a function $f: X \rightarrow X$ and $n \in \mathbb{N}$, by $f^{[n]}$ we mean

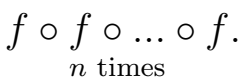

Given a metric space $(X, d)$, by:

- $P_{c p}(X)$ we mean the set of non-empty compact subsets of $X$

- the Hausdorff-Pompeiu metric we mean $H: P_{c p}(X) \times P_{c p}(X) \rightarrow[0,+\infty)$ given by

$$
H(A, B)=\max \{D(A, B), D(B, A)\}
$$

for all $A, B \in P_{c p}(X)$, where $D(A, B)=\sup _{x \in A}\left(\inf _{y \in B} d(x, y)\right)$ and $D(B, A)=$ $\sup _{x \in B}\left(\inf _{y \in A} d(x, y)\right)$

- a weakly Picard operator we mean a function $f: X \rightarrow X$ having the property that, for every $x \in X$, the sequence $\left(f^{[n]}(x)\right)_{n \in \mathbb{N}}$ is convergent to a fixed point of $f$. 


\section{Two results regarding the Hausdorff-Pompeiu metric}

Let us recall two very well known facts concerning the Hausdorff-Pompeiu metric.

Proposition 2.1. For a metric space $(X, d)$, we have

$$
H\left(\bigcup_{i \in I} K_{i}, \bigcup_{i \in I} L_{i}\right) \leq \sup _{i \in I} H\left(K_{i}, L_{i}\right),
$$

for every $\left(K_{i}\right)_{i \in I}$ and $\left(L_{i}\right)_{i \in I}$ finite families of elements from $P_{c p}(X)$.

Proposition 2.2. The metric space $\left(P_{c p}(X), H\right)$ is complete provided that the metric space $(X, d)$ is complete.

\section{More notations}

$B^{A}$ represents the set of functions from $A$ to $B$.

For a set $I$, we use the following notation:

- $I^{\{1,2, \ldots, n\}} \stackrel{\text { not }}{=} \Lambda_{n}(I)$; hence the elements of $\Lambda_{n}(I)$ can be written as words $\omega=\omega_{1} \omega_{2} \ldots \omega_{n}$ with $n$ letters from $I ; n$ is called the length of $\omega$ and is denoted by $|\omega|$.

- $\cup_{n \in \mathbb{N}} \Lambda_{n}(I) \stackrel{\text { not }}{=} \Lambda^{*}(I)$, where $\Lambda_{0}(I)$ consists on a single element, namely the empty word denoted by $\lambda$; hence $\Lambda^{*}(I)$ is the set of all finite words with letters from $I$.

Given a nonempty set $I, m, n \in \mathbb{N}$ and two words $\omega=\omega_{1} \omega_{2} \ldots \omega_{n} \in \Lambda_{n}(I)$ and $\theta=\theta_{1} \theta_{2} \ldots \theta_{m} \in \Lambda_{m}(I)$, by $\omega \theta$ we mean the concatenation of the words $\omega$ and $\theta$, i.e. $\omega \theta=\omega_{1} \omega_{2} \ldots \omega_{n} \theta_{1} \theta_{2} \ldots \theta_{m}$.

For a family of functions $\left(f_{i}\right)_{i \in I}$, where $f_{i}: X \rightarrow X$, and $\omega=\omega_{1} \omega_{2} \ldots \omega_{n} \in$ $\Lambda_{n}(I)$, we shall use the following notation: $f_{\omega} \stackrel{\text { not }}{=} f_{\omega_{1}} \circ f_{\omega_{2}} \circ \ldots \circ f_{\omega_{n}}$. By $f_{\lambda}$ we mean $I d_{X}$.

\section{The main result}

Definition 3.1. An iterated function system consisting of continuous functions satisfying Banach's orbital condition is described by:

- a complete metric space $(X, d)$

- a finite family of continuous functions $\left(f_{i}\right)_{i \in I}$, where $f_{i}: X \rightarrow X$, having the property that there exists $C \in(0,1)$ such that

$$
d\left(f_{\omega}(x), f_{\omega i}(x)\right) \leq C^{|\omega|} d\left(x, f_{i}(x)\right),
$$


for every $x \in X, i \in I$ and $\omega \in \Lambda^{*}(I)$.

We denote such a system by $\mathcal{S}=\left((X, d),\left(f_{i}\right)_{i \in I}\right)$.

Remark 3.1. Taking $\omega=j \in I$ and $i=j$ in the above definition we obtain that the functions $f_{i}$ satisfy Banach's orbital condition.

Definition 3.2. The fractal operator $F_{\mathcal{S}}: P_{c p}(X) \rightarrow P_{c p}(X)$, associated to the iterated function system consisting of continuous functions satisfying Banach's orbital condition $\mathcal{S}=\left((X, d),\left(f_{i}\right)_{i \in I}\right)$, is given by

$$
F_{\S}(K)=\cup_{i \in I} f_{i}(K)
$$

for every $K \in P_{c p}(X)$.

Definition 3.3. Every fixed point of the fractal operator associated to an iterated function system consisting of continuous functions satisfying Banach's orbital condition is called attractor of the system.

Lemma 3.1. Given an iterated function system consisting of continuous functions satisfying Banach's orbital condition $\mathcal{S}=\left((X, d),\left(f_{i}\right)_{i \in I}\right)$, we have

$$
H\left(F_{\mathcal{S}}^{[n]}(\{x\}), F_{\mathcal{S}}^{[n+1]}(\{x\})\right) \leq C^{n} H\left(\{x\}, F_{\mathcal{S}}(\{x\})\right),
$$

for every $x \in X$ and every $n \in \mathbb{N}$.

Proof. On the one hand, we have

$$
\begin{gathered}
D\left(F_{\mathcal{S}}^{[n]}(\{x\}), F_{\mathcal{S}}^{[n+1]}(\{x\})\right)=\sup _{\omega \in \Lambda_{n}(I)}\left(\inf _{\theta \in \Lambda_{n+1}(I)} d\left(f_{\omega}(x), f_{\theta}(x)\right)\right)= \\
=\sup _{\omega \in \Lambda_{n}(I)}\left(\inf _{v \in \Lambda_{n}(I),} d\left(f_{\omega}(x), f_{v i}(x)\right)\right) \leq \sup _{\omega \in \Lambda_{n}(I)}\left(\inf _{i \in I} d\left(f_{\omega}(x), f_{\omega i}(x)\right)\right) \stackrel{\text { Definition } 3.1}{\leq} \\
\leq C^{n_{i n f}} \inf _{i \in I} d\left(x, f_{i}(x)\right)=C^{n} D\left(\{x\}, F_{\mathcal{S}}(\{x\})\right)
\end{gathered}
$$

for every $x \in X$ and every $n \in \mathbb{N}$.

On the other hand, we have

$$
\begin{gathered}
D\left(F_{\mathcal{S}}^{[n+1]}(\{x\}), F_{\mathcal{S}}^{[n]}(\{x\})\right)=\sup _{\theta \in \Lambda_{n+1}(I)}\left(\inf _{\omega \in \Lambda_{n}(I)} d\left(f_{\theta}(x), f_{\omega}(x)\right)\right)= \\
=\sup _{\substack{v \in \Lambda_{n}(I), i \in I}}\left(\inf _{\omega \in \Lambda_{n}(I)} d\left(f_{v i}(x), f_{\omega}(x)\right)\right) \leq \sup _{\substack{v \in \Lambda_{n}(I) \\
i \in I}}\left(d\left(f_{v i}(x), f_{v}(x)\right)\right) \stackrel{\text { Definition } 3.1}{\leq} \\
\leq C^{n} \sup _{i \in I}\left(d\left(f_{i}(x), x\right)\right)=C^{n} D\left(F_{\mathcal{S}}(\{x\}),\{x\}\right)
\end{gathered}
$$

for every $x \in X$ and every $n \in \mathbb{N}$.

From (1) and (2) we obtain the conclusion. 
Theorem 3.2. Every iterated function system consisting of continuous functions satisfying Banach's orbital condition has attractor. More precisely, the fractal operator associated to such a system is a weakly Picard operator.

Proof. Let $\mathcal{S}=\left((X, d),\left(f_{i}\right)_{i \in I}\right)$ be an iterated function system consisting of continuous functions satisfying Banach's orbital condition.

Now, let us fix $K_{0} \in P_{c p}(X)$ and, for every $n \in \mathbb{N}$, denote $F_{\mathcal{S}}^{[n]}\left(K_{0}\right)$ by $K_{n}$.

Claim 1. The sequence $\left(K_{n}\right)_{n \in \mathbb{N}}$ is Cauchy.

Justification of claim 1. We have

$$
\begin{gathered}
H\left(K_{n}, K_{n+1}\right)=H\left(F_{\mathcal{S}}^{[n]}\left(K_{0}\right), F_{\mathcal{S}}^{[n+1]}\left(K_{0}\right)\right)= \\
=H\left(\cup_{x \in K_{0}} F_{\mathcal{S}}^{[n]}(\{x\}), \cup_{x \in K_{0}} F_{\mathcal{S}}^{[n+1]}(\{x\})\right) \stackrel{\text { Proposition 2.1 }}{\leq} \\
\leq \sup _{x \in K_{0}} H\left(F_{\mathcal{S}}^{[n]}(\{x\}), F_{\mathcal{S}}^{[n+1]}(\{x\})\right) \stackrel{\text { Lemma } 3.1}{\leq} \\
\leq C^{n} \sup _{x \in K_{0}} H\left(\{x\}, F_{\mathcal{S}}(\{x\})\right)=C^{n} M_{K_{0}},
\end{gathered}
$$

for every $n \in \mathbb{N}$, where $M_{K_{0}} \stackrel{\text { not }}{=} \sup _{x \in K_{0}} H\left(\{x\}, F_{\mathcal{S}}(\{x\})\right)$. Note that, from continuity reasons, $M_{K_{0}} \in \mathbb{R}$. The fact that $C \in(0,1)$ and (1) imply that the sequence $\left(K_{n}\right)_{n \in \mathbb{N}}$ is Cauchy and this closes the justification of Claim 1.

Proposition 2.2 and Claim 1 assure the existence of a set $A_{K_{0}} \in P_{c p}(X)$ such that $\lim _{n \rightarrow \infty} K_{n}=A_{K_{0}}$, i.e.

$$
\lim _{n \rightarrow \infty} F_{\mathcal{S}}^{[n]}\left(K_{0}\right)=A_{K_{0}} .
$$

Claim 2. $F_{\mathcal{S}}$ is sequentially continuous.

Justification of claim 2. Using the same technique as the one used in Theorem 3.2 Fact 1 , from [16] one can prove that

$$
\lim _{n \rightarrow \infty} H\left(Y_{n}, Y\right)=0 \Rightarrow \lim _{n \rightarrow \infty} H\left(F_{\mathcal{S}}\left(Y_{n}\right), F_{\mathcal{S}}(Y)\right)=0,
$$

for every $\left(Y_{n}\right)_{n \in \mathbb{N}} \subseteq P_{c p}(X)$ and $Y \in P_{c p}(X)$.

Claim 3. $A_{K_{0}}$ is a fixed point of $F_{\delta}$.

Justification of claim 3. Using (2) and Claim 2, we get

$$
\lim _{n \rightarrow \infty} F_{\mathcal{S}}^{[n+1]}\left(K_{0}\right)=F_{\mathcal{S}}\left(A_{K_{0}}\right)
$$

From (2) and (3) we conclude that $F_{\mathcal{S}}\left(A_{K_{0}}\right)=A_{K_{0}}$, i.e. $A_{K_{0}}$ is an attractor of $\mathcal{S}$, and the justification of Claim 3 is done.

Claim 3 and $(2)$ show that the operator $F_{\S}$ is weakly Picard. 
Corollary 3.3. Given a complete metric space $(X, d)$, every continuous function $f: X \rightarrow X$ satisfying Banach's orbital condition is a weakly Picard operator.

Proof. Just consider in Theorem 3.2 the case when the set $I$ has one element and take into account Remark 3.1.

\section{Examples}

A. Let us consider the complete metric spaces $(X, d)$ and $(Y, \rho)$ and the finite family of continuous functions $\left(g_{i}\right)_{i \in I}$, where, for every $i \in I$, the function $g_{i}: X \times Y \rightarrow X$ has the property that there exists $C_{i} \in[0,1)$ such that

$$
d\left(g_{i}(x, y), g_{i}\left(x^{\prime}, y\right)\right) \leq C_{i} d\left(x, x^{\prime}\right),
$$

for every $x, x^{\prime} \in X$ and $y \in Y$.

Then we can consider the family of continuous functions $\left(f_{i}\right)_{i \in I}$, where for every $i \in I$, the function $f_{i}: X \times Y \rightarrow X \times Y$ is given by

$$
f_{i}(x, y)=\left(g_{i}(x, y), \pi_{2}(x, y)\right),
$$

for every $(x, y) \in X \times Y$.

In the sequel we adopt the notation $C \stackrel{\text { not }}{=} \max _{i \in I} C_{i}$ and we endow $X \times Y$ with the metric $d_{\max }$ given by $d_{\max }\left(\left(x_{1}, y_{1}\right),\left(x_{2}, y_{2}\right)\right)=\max \left\{d\left(x_{1}, x_{2}\right), \rho\left(y_{1}, y_{2}\right)\right\}$ for every $\left(x_{1}, y_{1}\right),\left(x_{2}, y_{2}\right) \in X \times Y$.

\section{Claim 4.1.}

$$
d_{\max }\left(f_{\omega}(x, y), f_{\omega i}(x, y)\right) \leq C^{|\omega|} d_{\max }\left((x, y), f_{i}(x, y)\right),
$$

for every $i \in I, \omega \in \Lambda^{*}(I)$ and $(x, y) \in X \times Y$.

Justification of the claim. Since

$$
f_{i_{1} i_{2} \ldots i_{n}}(x, y)=\left(g_{i_{1}}\left(g_{i_{2}}\left(\ldots\left(g_{i_{n}}(x, y), y\right)\right) \ldots, y\right), y\right)
$$

and

$$
f_{i_{1} i_{2} \ldots i_{n} i}(x, y)=\left(g_{i_{1}}\left(g_{i_{2}}\left(\ldots\left(g_{i_{n}}\left(\left(g_{i}(x, y), y\right), y\right), y\right)\right) \ldots, y\right), y\right),
$$

based on (1), we get

$$
d_{\max }\left(f_{i_{1} i_{2} \ldots i_{n}}(x, y), f_{i_{1} i_{2} \ldots i_{n} i}(x, y)\right) \leq C^{n} d\left(x, g_{i}(x, y)\right)=
$$




$$
=C^{n} d_{\max }\left((x, y),\left(g_{i}(x, y), y\right)\right)=C^{n} d_{\max }\left((x, y), f_{i}(x, y)\right),
$$

for every $n \in \mathbb{N}, i_{1}, i_{2}, \ldots, i_{n}, i \in I$ and $(x, y) \in X \times Y$. The justification of the claim is done.

The claim assures us that $\mathcal{S}=\left(\left(X \times Y, d_{\max }\right),\left(f_{i}\right)_{i \in I}\right)$ is an iterated function system consisting of continuous functions satisfying Banach's orbital condition.

Now we present an attractor of $\mathcal{S}$.

We start by noting that, for an arbitrary but fixed $y \in Y$, taking into account (1), we can consider the classical iterated function system (i.e consisting of Banach contractions - see [11]), denoted by $\mathcal{S}_{y}$, described by the complete metric space $(X, d)$ and by the finite family of functions $\left(h_{i}\right)_{i \in I}$, where $h_{i}$ : $X \rightarrow X$ is given by $h_{i}(x)=g_{i}(x, y)$ for every $x \in X$. Then $\mathcal{S}_{y}$ has a unique attractor which will be denoted by $A_{y} \in P_{c p}(X)$ (this means that $A_{y}$ is the unique fixed point of $F_{\mathcal{S}_{y}}: P_{c p}(X) \rightarrow P_{c p}(X)$ given by $F_{\mathcal{S}_{y}}(K)=\cup_{i \in I} h_{i}(K)$ for every $\left.K \in P_{c p}(X)\right)$. We continue by remarking that, for $K^{*}=\{x\} \times$ $K \in P_{c p}(X \times Y)$, where $x \in X$ and $K \in P_{c p}(Y)$, we have $F_{\mathcal{S}}^{[n]}\left(K^{*}\right)=$ $\cup_{y \in K} F_{\delta_{y}}^{[n]}(\{x\}) \times\{y\}$ for every $n \in \mathbb{N}$. As $\lim _{n \rightarrow \infty} F_{\delta_{y}}^{[n]}(\{x\})=A_{y}$, we conclude that $\lim _{n \rightarrow \infty} F_{\mathcal{S}}^{[n]}\left(K^{*}\right)=\cup_{y \in K} A_{y} \times\{y\}$, so $\underset{y \in K}{\cup} A_{y} \times\{y\}$ is an attractor of $\mathcal{S}$.

B. Let us consider two continuous functions $g_{1}, g_{2}:[0,1] \rightarrow \mathbb{R}$ such that $g_{1} \leq 0 \leq 1 \leq g_{2}, \Gamma_{g_{1}, g_{2}}=\left\{(x, y) \in \mathbb{R}^{2} \mid x \in[0,1]\right.$ and $\left.y \in\left[g_{1}(x), g_{2}(x)\right]\right\}$ and the continuous functions $f_{1}, f_{2}: \Gamma_{g_{1}, g_{2}} \rightarrow \Gamma_{g_{1}, g_{2}}$ given by

$$
f_{1}(x, y)=\left(x, \frac{1}{3} y\right) \text { and } f_{2}(x, y)=\left(x, \frac{1}{3} y+\frac{2}{3}\right),
$$

for every $(x, y) \in \Gamma_{g_{1}, g_{2}}$.

Using the same arguments as the ones used in the previous example, one can easily check that

$$
d\left(f_{\omega}(u), f_{\omega i}(u)\right) \leq \frac{1}{3^{|\omega|}} d\left(u, f_{i}(u)\right)
$$

for every $u \in \Gamma_{g_{1}, g_{2}}, i \in\{1,2\}$ and $\omega \in \Lambda^{*}(\{1,2\})$, so

$$
\mathcal{S}=\left(\left(\Gamma_{g_{1}, g_{2}}, d_{\max }\right),\left(f_{i}\right)_{i \in\{1,2\}}\right)
$$

is an iterated function system consisting of continuous functions satisfying Banach's orbital condition. One can easily check that $\lim _{n \rightarrow \infty} F_{\mathcal{S}}^{[n]}(K)=\pi_{1}(K) \times$ $C$, for every $K \in P_{c p}\left(\Gamma_{g_{1}, g_{2}}\right)$, so $\pi_{1}(K) \times C$ is an attractor of $\mathcal{S}$, where $C$ is the Cantor ternary set.

Remark 4.1. For the above mentioned examples the operator $F_{\mathcal{S}}$ is weakly Picard but it is not Picard. 


\section{$5 \quad$ Further lines of research}

We end this paper by mentioning some further lines of research. Namely, given an iterated function system $\mathcal{S}=\left((X, d),\left(f_{i}\right)_{i \in I}\right)$ consisting of continuous functions satisfying Banach's orbital condition, we are going to:

- find supplementary conditions under which $F_{\mathcal{\delta}}$ is Picard operator

- define and study the properties of an analogue of the canonical projection from the shift space to $A_{K_{0}}$, where $K_{0} \in P_{c p}(X)$

- define and study the properties of an analogue of the Hutchinson measure associated to the system $\mathcal{S}$ endowed with a system of probabilities.

\section{References}

[1] J. Andres, M. Rypka, Multivalued fractals and hyperfractals, Internat. J. Bifur. Chaos Appl. Sci. Engrg. 22 (2012), DOI 10.1142/S02181127412500095.

[2] M. Barnsley, K., Leśniak, M. Rypka, Chaos game for IFSs on topological spaces, J. Math. Anal. Appl. 435 (2016), 1458-1466.

[3] V. Berinde, Iterative approximation of fixed points, Lecture Notes in Mathematics 1912, Springer, Berlin, 2007.

[4] M. Boriceanu, M. Bota, A. Petruşel, Multivalued fractals in b-metric spaces, Cent. Eur. J. Math. 8 (2010), 367-377.

[5] C. Chifu, A. Petruşel, Multivalued fractals and generalized multivalued contractions, Chaos Solitons Fractals 36 (2008), 203-210.

[6] D. Dumitru, Attractors of infinite iterated function systems containing contraction type functions, An. Ştiinţ. Univ. Al. I. Cuza Iaşi, Mat. N.S., 59 (2013), 281-298.

[7] F. Georgescu, IFSs consisting of generalized convex contractions, $A n$. Stiint. Univ. "Ovidius" Constanţa, Ser. Mat., 25 (2017), 77-86.

[8] F. Georgescu, R. Miculescu, A. Mihail, Iterated function systems consisting of $\varphi$-max-contractions have attractor, in print J. Fixed Point Theory Appl., available at arXiv:1704.02652

[9] G. Gwóźdź-Lukowska, J. Jachymski, IFS on a metric space with a graph structure and extensions of the Kelisky-Rivlin theorem, J. Math. Anal. Appl. 356 (2009), 453-463. 
[10] T. L. Hicks, B. E. Rhoades,A Banach type fixed point theorem, Math. Japonica 24 (1979), 327-330.

[11] J. E. Hutchinson, Fractals and self similarity, Indiana Univ. Math. J. 30 (1981), 713-747.

[12] L. Ioana, A. Mihail, Iterated function systems consisting of $\varphi-$ contractions, Results Math. 72 (2017), 2203-2225.

[13] A. A. Ivanov, Fixed points of metric space mappings (in Russian), Isledovaniia po topologii.II, Akademia Nauk, Moskva, 1976, 5-102.

[14] E. Llorens-Fuster, A. Petruşel, J.-C. Yao, Iterated function systems and well posedness, Chaos Solitons Fractals 41 (2009), 1561-1568.

[15] L. Máté, The Hutchinson-Barnsley theory for certain noncontraction mappings, Period. Math. Hungar. 27 (1993), 21-33.

[16] R. Miculescu, A. Mihail, Reich-type iterated function systems, J. Fixed Point Theory Appl. 18 (2016), 285-296.

[17] R. Miculescu, A. Mihail, A generalization of Istrățescu's fixed point theorem for convex contractions, Fixed Point Theory 18 (2017), 689-702.

[18] T. Nazir, S. Silvestrov, M. Abbas, Fractals of generalized FHutchinson operator, Waves Wavelets Fractals Adv. Anal. 2 (2016), 2940 .

[19] A. Petruşel, Iterated function system of locally contractive operators, Rev. Anal. Numér. Théor. Approx. 33 (2004), 215-219.

[20] A. Petruşel, A. Soos, Self-similar sets and fractals generated by Ćirić type operators, J. Nonlinear Sci. Appl. 8 (2015), 1048-1058.

[21] I. A. Rus, Some fixed point theorems in metric spaces, Rend. Ist. Matem. Univ. Trieste 3 (1971), 169-172.

[22] I. A. Rus, On the method of successive approximations (in Russian), Revue Roum. Math. Pures Appl. 17 (1972), 1433-1437.

[23] D. R. Sahu, A. Chakraborty, R. P. Dubey, K-iterated function system, Fractals 18 (2010), 139-144.

[24] N. A. Secelean, Iterated function systems consisting of $F$-contractions, Fixed Point Theory Appl. (2013), 2013:277.

[25] M. Taskovic, Osnove teorije fiksne tacke (Fundamental Elements of Fixed Point Theory), Matematicka biblioteka 50, Beograd, 1986. 
[26] N. Van Dung, N. Petruşel, A. Petruşel, On iterated function systems consisting of Kannan maps, Reich maps, Chatterjea type maps, and related results, J. Fixed Point Theory Appl. 19 (2017), 2271-2285.

Radu Miculescu

Faculty of Mathematics and Computer Science

Transilvania University of Braşov

Iuliu Maniu Street, nr. 50500091 Braşov Romania

E-mail: miculesc@yahoo.com

Alexandru Mihail

Faculty of Mathematics and Computer Science

Bucharest University, Romania

Str. Academiei 14, 010014, Bucharest

E-mail: mihail_alex@yahoo.com

Irina Savu

Faculty of Mathematics and Computer Science

Bucharest University, Romania

Str. Academiei 14, 010014, Bucharest, Romania

E-mail: irinamsavu@yahoo.com 